\title{
Eye-tracking Web Usability Research
}

\author{
Paweł Weichbroth, Krzysztof Redlarski, Igor Garnik \\ Gdańsk University of Technology \\ Faculty of Management and Economics \\ Department of Applied Informatics in Management \\ ul. Narutowicza 11/12 80-233 Gdańsk, Poland \\ Email: pawel.weichbroth, krzysztof.redlarski, igor.garnik@zie.pg.gda.pl
}

\begin{abstract}
In this paper we present the results of a study that aims to evaluate the usability of three selected web services, based on eye-tracking and thinking aloud techniques. The gathered comments and observations, recapitulated and supported by particular measures, allow us to discover and describe typical user behavior pertaining to given tasks to solve.
\end{abstract}

\section{INTRODUCTION}

$\mathrm{W}$ EB portals are one of the main sources of information and news. These were some of the first information services that nearly a quarter of a century ago appeared on the Internet. Initially, they delivered information in a similar way to newspapers, but they also contained links to other subject-related services. However, the most important role of a portal is still to provide timely and reliable information, relevant to Internet users' needs. Unfortunately, as shown by the results of the research presented in this paper, users often have trouble finding even basic information that, due to the nature of the service, should be available there.

It may be noted that Internet users seek information in two ways: if the type of information is sought occasionally (e.g. only once), then they use a search engine, such as Bing, Google or Yahoo [1]. In contrast, when they frequently seek information of the same type (e.g. exchange rates on the current day), then they use proven and trusted web services, such as Internet portals [2].

Over the years, we can observe how, under the influence of development of technology and design trends, the appearance of web pages and the way information is exhibited is changing. User behavior patterns of searching for information are changing as well. These patterns are affected by many factors (which enforces a different way of seeking information), e.g. the more intensive use of mobile devices, the age or even the gender of users [3, 4]. It was the basis for the research enabling the observation and description of patterns that can be found among current users, and also the determination of the reasons why access to some types of information is difficult (or even unavailable) and how to prevent this.

\section{RELATED WORKS}

Montero et al. [5] showed that the essence of design patterns is to capture the design experience in such a form which can be used effectively and repeatedly. However, adapting patterns is not a straightforward task, because the designer must demonstrate expertise and flexibility in the design process and operate on a high level of abstraction. The authors proposed conceptualizing web design pattern knowledge into the form of an ontology. The depicted generic hypermedia model, which describes the structure of a hypermedia application, includes such elements as: node (an information holder, e.g. a web page, a frame, a pop-up window), content (a piece of information, e.g. a text, a binary file or an executable application), link (a connection between two or more nodes or contents) and anchor (a source or target reference to an internal or external content or node). Design pattern format, usually expressed in natural language, is typically specified by such attributes as: name (an unambiguous identifier), category (used to classify the pattern body based on several criteria, like purpose or scope), problem (outlines the adaption scenario), solution (outlines the desired outcome) and related-patterns (depicts equivalents).

Based on Jakobson's communication model, Thorlacius [6] introduced and elucidated a visual communication model, where web-specific aspects in terms of navigation and interaction were taken into account. The model consists of the six following factors: product, context, medium, code, the addresser (actual or implicit) and the addressee (actual or implicit). The product is both the content and the form, along with two communication functions: formal and sublime aesthetic. The first one is the concept of visual symbols, considered in terms of colors, illustrations, typography and design in accordance with modern conventions of website layout; it reflects user experiences that contribute to "good look and feel". The second function is the question of when and where to use visual elements like flash animations and expressive illustrations, and whether innovative design should be applied; it arises from "the space between the known and the unknown" and is harder to describe in detail. The context refers to the featured (core) 
content of the product. The medium is the connecting link between the addresser and the addressee in order to establish a communication channel. The code is a system of signs where each unique sequence returns a different meaning, which is presumed known to both sides. The addresser is a single person or persons responsible for the content being published on the website, where the actual is the only person who speaks for himself about the real intentions which lay behind the website, and the implicit is directly personified by visible means on the website. Finally, the last factor in the cited model is the addressee, who is the content receiver, who can actually experience the product (actual) or who can be identified through an appearance analysis (implicit).

\section{EXPERIMENTAL SETUP}

\section{A. Participants}

43 participants were involved in the experiment: 21 males and 22 females, with the age average of 22.53 , all students of the Gdansk University of Technology, the Faculty of Management and Economics.

\section{B. Apparatus}

In our research, eye movements (saccades, fixations, pupil diameter) were recorded with the infrared camera-based Tobii TX300 eye-tracker system, where the light source and camera are permanently affixed to a monitor. It has a $300 \mathrm{~Hz}$ sampling frequency, and the tracking technique is "dark pupil". We used Tobii Studio software working under MS Windows7 (x64).

During the experiment the voice conversation between the moderator and participant was recorded and we made handwritten short notes of comments and remarks, and evidenced the results of tasks given to solve. Tobii Studio allowed us only to use Internet Explorer as a web browser, from this reason we were not able to examine other web browsers.

\section{Objects and stimuli}

The set of objects consists of three different web portals that bring information together from diverse topics and sources in a uniform way. Onet and Wiadomosci24 are the most recognizable Polish-language web portals in Poland, along with the English-language BBC. All of them are information-aware, where up-to-date news, gossip and advertisements play a major role. The context surrounding the examined websites is largely the same, where the top three mainstream contexts can be distinguished: political, social and economic. However, they differ significantly if the context scope is taken into consideration. The addressers are a group of people involved in developing, maintaining and promoting particular sections of a website, in cooperation with journalists that comment on the current information stream, carry out interviews, and report events and facts on the site. The addressees are generally users who purposely request access to website resources, using a suitable electronic device (e.g. personal computer, tablet or smart phone).

\section{D.Procedure}

The procedure consisted of 18 steps (Fig. 1), including 8 instructions (I), 3 questions (Q) and 6 tasks (T). The first instruction (I1) was a welcome screen and briefly described the purpose of the research. Next, in three questions (Q1-Q3) we asked the participant respectively about their sex, age and English language skills. Each of the second to the seventh instructions (I2-I7), preceded and described the subsequent tasks (T1-T6), and the last instruction (I8) was an acknowledgment of participation.

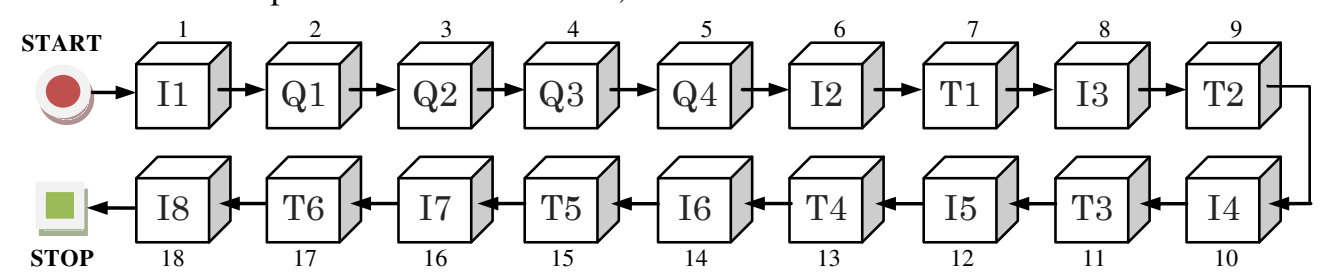

Fig. 1. The research procedure sequence

There were two separate tasks defined: the first was to find the current weather in Gdansk and the second was to find the exchange rate of Polish currency to the Euro. The maximum time allowed per task was 60 seconds. Tasks T1 and $\mathrm{T} 2$ were performed on Onet, $\mathrm{T} 3$ and $\mathrm{T} 4$ on the $\mathrm{BBC}$ site and T5 and T6 on Wiadomosci24. We decided to use the thinking aloud technique during the test to help in understanding the manner of each participant's behavior while performing the task.

\section{E. Eye movement analysis}

A quite apparent assumption laid the foundation-stone for the concept of areas of interest: informative regions of a scene receive more of an observer's attention than the less informative regions $d o$. In this context, the qualifier "informative" reflects the degree of how much that particular area contributes to the understanding (perception) of the scene of sight from which the area is taken. However, a separation of such areas still poses many challenges for the development and research community. In Tobii Studio, AOIs are created by drawing ellipses, rectangles or polygons over any type of stimuli to get statistics for eye-tracking metrics for one or more recordings. 


\section{RESUlTS}

Quantitative and qualitative methods to collect data were concurrently used that respectively correspond to the eyetracker device and thinking aloud technique. The quantitative analysis (heat map method) helped to identify the main areas of the participants' interest which drew their attention the most.

The analysis of the Onet service (Fig. 2) shows the largest concentration of the participants' sight on the information in the upper right corner of the site. The influencing factor for this might be the fact that the participants declared the greatest knowledge of this web service.

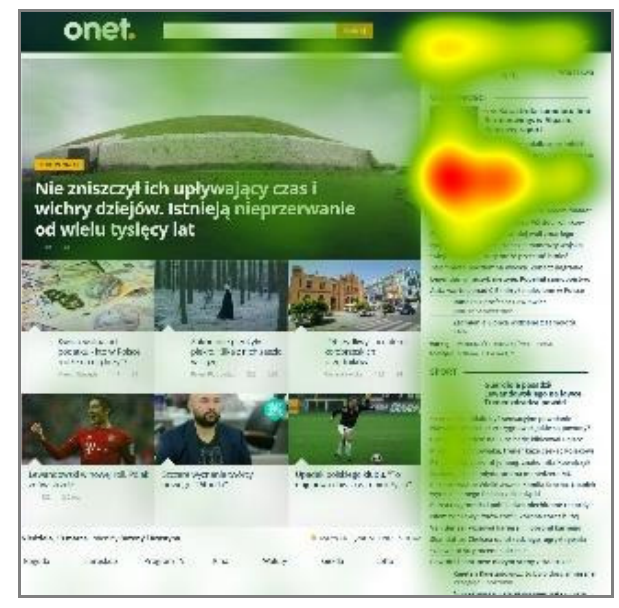

Fig. 2 The Onet heat map

In case of the BBC service (Fig. 3) we observed an increased interest in the upper part of the service. This was the location of the menu bar which was thoroughly followed by the participants.

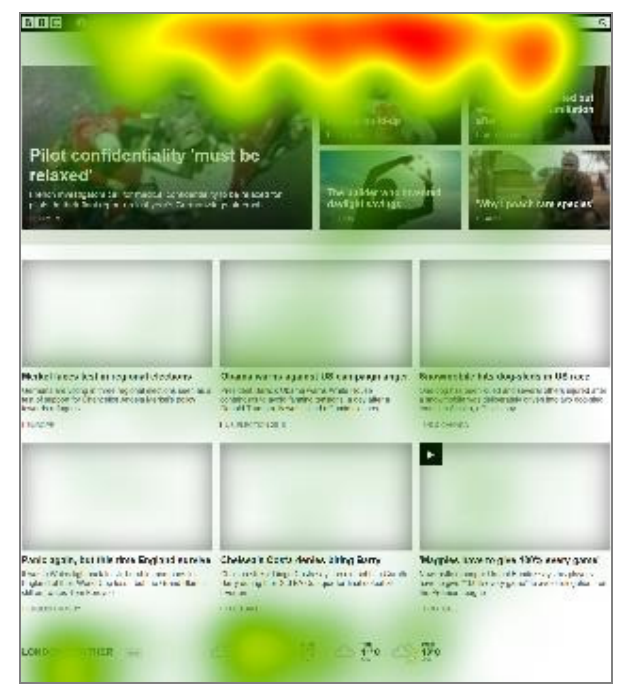

Fig. 3. The BBC heat map

Interestingly, the participants also declared the lowest knowledge of the BBC service. This means that their behavior was the most natural and intuitive, and that there were no disturbance factors, such as prior acquaintance with the site.

The analysis of the Wiadomosci24 heat map (Fig. 4) allows for the observance of an increased concentration on the largest graphic and a field in the upper right corner of the service. The interest in the field (in the upper right corner) could result from the order of task execution.

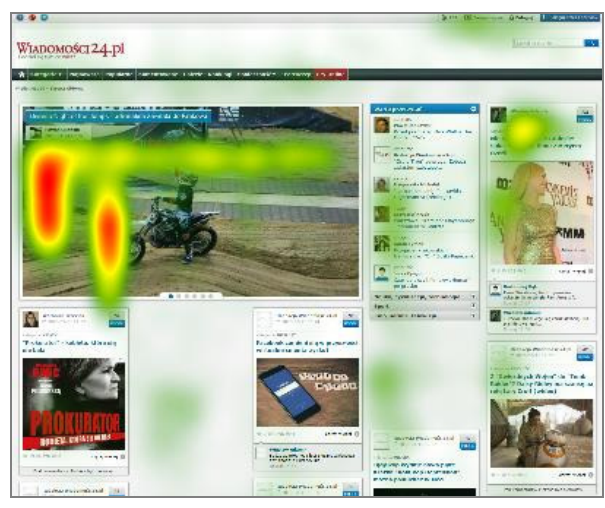

Fig. 4. The Wiadomosci24 heat map

In this case the participants sought required information in the similar areas like in the case of Onet service. Such behavior was probably a result of prior connotations and users' experience with previously tested websites.

In addition, the quantitative analysis involved the reconstruction of video records and the verification of handwritten notes from the study. The data analysis helped to identify three dominant patterns of behavior among users in terms of how they try to reach the necessary information:

- search tool, a method based on finding the website search tool, which leads the user to the desired information by entering a user keyword;

- menu bar, a method based on finding the options bar or toolbar; analogically to the software application, the user tried to use a systematized and categorized tool (e.g. the menu bar), which should lead the user to the desired information;

- home page, a method based on searching the website's home page content for information; the user that decided to search the information service expected that they would find information on the home page.

Table I shows how often the particular method of

TABLE I

The Results Summary Of The CONDucted ObSeRvations

\begin{tabular}{|l|c|c|c|c|c|c|c|}
\hline $\begin{array}{c}\text { Method of } \\
\text { search } \\
\text { information }\end{array}$ & \multicolumn{2}{|c|}{ Onet } & \multicolumn{2}{|c|}{ BBC } & \multicolumn{2}{c|}{$\begin{array}{c}\text { Wiadomosci } \\
\text { 24 }\end{array}$} & \multirow{2}{*}{ AVG } \\
\cline { 2 - 7 } & T1 & T2 & T1 & T2 & T1 & T2 & \\
\hline Search tool & 2 & 5 & 25 & 19 & 27 & 20 & 16.3 \\
\hline Menu bar & 15 & 25 & 30 & 42 & 39 & 40 & 31.8 \\
\hline Home page & 41 & 42 & 39 & 40 & 39 & 37 & 39.7 \\
\hline $\begin{array}{l}\text { Number of } \\
\text { participants }\end{array}$ & 43 & 43 & 43 & 43 & 43 & 43 & - \\
\hline
\end{tabular}


information search were used in the particular web service. The results of the first task showed the differences among the participants in the selection sequence of a particular model of behavior. Some of the participants decided to seek information through the menu bar, some by analyzing the contents of the home page, and some by using the search tool (both available in the information service, as well as a built-in web browser). Such behavior patterns may have many origins: previous experience in use of the particular service or knowledge of the language in which the content is presented (the participants declared different level of English language skills). These are certainly factors requiring further research on their impact on the behavior of users of information services in their search for desired information.

In the second stage of the analysis of the results of these studies, information for previously identified ways to search was verified. For this purpose, we defined areas of interest (AOI) for each of the services. They included a specific part of the site which contained information belonging to one of three identified groups ("search tool", "menu bar", "index/home page").

The quantitative analysis of models of user behavior, in the context of the search for information in each individual information service, was carried out using the following variables: time to first fixation, fixations before the participant appears in the AOI, fixation duration, fixation count, visit duration, total visit duration, percentage fixated, and percentage clicked.

An analysis showed significant differences regarding the usability of the home site, as well as allowed to identify changes in the behavior of tested users, depending on the home page services. In the case of the Wiadomosci24 service the study participants focused their sight on a twocolumn layout. The structure of other services resulted in a single-column data analysis. The reason for this and no other particular behavior was probably the difference in the system frame, which in the case of Wiadomości24 was more diffuse. The participants enjoyed situations when sought information was displayed directly on the screen and it did not require the user to additionally scroll through. However, the lower the content was on the home site, the decreasing level of interest was observed. The Wiadomosci24 service was a kind of exception. In this case, the participants analyzed the content available at the bottom of the website for much longer. The reason for this was probably the form that resembles the structure of a typical "toolbar menu". For the rest of the websites there was the same trend (as in the case of the Onet and BBC services) - the lower the position of the content, the less the interest of the user.

\section{V.CONCLUSIONS}

The aim of the study was to analyze the typical behavior of users of web services in the search for information. The research helped to identify not only common patterns and behaviors that occurred among users. The first stage of the analysis of the study results allowed typical patterns to be identified, which consisted in an attempt to find the desired information through the search tool, the menu bar, or an analysis of the home page. Then quantitative analysis confirmed the foundations of the first stage of data analysis and identified probable causes of the different behavior of study participants.

All participants completed the first three tasks, whereas none of them were able to complete the last three. We purposely designed a set of tasks in such a manner where the first part was solvable while the second was unsolvable. Such a scenario aimed to stimulate participants to comment on obstacles and constraints, which eventually led to evaluate the usability of a particular service on the one hand, while on the other hand, random surfing progressively and subconsciously increased their concentration and cognition in order to solve given tasks. In consequence, we observed different kind of reactions: surrender (task abandonment after a few failed attempts), impatience (increasing tension and distraction over time) and self-inventiveness (out-of-the box actions, i.e. taking advantage of web browser functionality, opening new tab windows, simultaneously using well-known search engines).

To the best of our knowledge, eye-tracking analysis combined with the thinking aloud technique can provide valuable guidance to designers on the construction of information services and a starting point for further refinement of usability. In the near future, we plan to evaluate different structure patterns of websites and investigate the scale of interactions between three different groups of artefacts (i.e. standards, interface schema and data flow diagrams) in order to embody modifications included in subsequent prototypes, developed in Python. Moreover, we will verify the usefulness of the evaluation matrix template, introduced in [7].

\section{REFERENCES}

[1] B. Sparrow, J. Liu, and D.M. Wegner, Google effects on memory: cognitive consequences of having information at our fingertips. Science, vol. 333(6043), 2011, pp. 776-778.

[2] E. Go, K. H. You, E. Jung, and H. Shim H, Why do we use different types of websites and assign them different levels of credibility? Structural relations among users' motives, types of websites, information credibility, and trust in the press. Computers in Human Behavior, vol. 54, 2016, pp. 231-239.

[3] S.-Y. Chen, and J.-Y. Tzeng, College female and male heavy internet users' profiles of practices and their academic grades and psychosocial adjustment. Cyberpsychology, Behavior and Social Networking, vol. 13(3), 2010, pp. 257-62.

[4] J. Pokrywczynski, and J. Wolburg, A psychographic analysis of Generation Y college students. Journal of Advertising Research, 2001, pp. 33-52.

[5] S. Montero, P. Díaz, and O. Aedo, Formalization of web design patterns using ontologies. In: Advances in Web Intelligence. Springer, Berlin 2003, pp. 179-188.

[6] L. Thorlacius, Visual Communication in Web Design - Analyzing Visual Communication. In: International Handbook of Internet Research, Springer, 2010, pp. 455-476.

[7] P. Weichbroth, and M. Sikorski, User Interface Prototyping. Techniques, Methods and Tools. Studia Ekonomiczne. Zeszyty Naukowe Uniwersytetu Ekonomicznego w Katowicach, 2015, pp. 184-198. 